\title{
Study of Cyclic Constrained Groove Pressing Factors on Hardness Behavior of Al/Sic - MMC Subjected to Severe Plastic Deformation
}

\author{
H.S.Siddesha ${ }^{1}$, M.Shantharaja ${ }^{2}$ \\ ${ }^{1}$ ACS College of Engineering, Department of Mechanical Engineering, Bangalore-560074.India \\ ${ }^{2}$ UVCE, Dept. of Mechanical Engineering, K.R. Circle, Bangalore University Bangalore-560001.India
}

\begin{abstract}
Cyclic constrained groove pressing (CCGP) are widely used for the production of an ultra finegrained, Nano/sub microcrystalline structure in industries to compensate the high strength metal plates components used in automobiles. The present paper focus on The mechanical properties in nanostructure $\mathrm{Al} / \mathrm{Sic}$ composite plate processed by a new technique, cyclic constrained groove pressing (CCGP), were studied using micro hardness tester. Taguchi technique and the analysis of variance (ANOVA) techniques were carried out to investigate the degree of importance of CCGP process parameters i.e. Percentage of Sic, strain rate, number of passes, plate thickness and heat treatment for four different levels. The results indicated that the percentage of Sic factor has a major influence on the hardness of the composite plate, followed by no. of passes, Heat treatment, plate thickness and strain rate. The Taguchi method and the ANOVA technique was an effective tool to predict the degree of importance of the CCGP parameters on micro hardness CGP specimens.
\end{abstract}

Key words: - Severe plastic deformation, CCGP, Al/SiC composites, Taguchi method, Micro hardness.

\section{INTRODUCTION}

The process of severe plastic deformation (SPD) is gaining great interest in material science because it is useful to refine microstructures to the sub micrometer or nanometer levels [1]. In $\mathrm{Al} / \mathrm{Sic}$ composites,it is generally difficult to reduce the grain size below $10 \mu \mathrm{m}$ through the conventional recrystallization process following thermo-mechanical treatments. This difficult arises from the inherent nature of $\mathrm{Al} / \mathrm{Sic}$ composites that the staking fault energy is relatively large so that it is easy for the recovery of dislocation to occur [2]. One of these advantages is a capability of producing large samples that are free from any residual porosity and readily amenable to mechanical testing and forming operations[3]. Among the various SPD techniques proposed most of the methods are intended for processing bulk materials; very few methods like accumulative[4,5],repetitive corrugation and straightening(RCS)[8,9]and cyclic constrained groove pressing(CCGP) [6,7] are capable of processing sheet materials. CCGP process was invented recently and it is promising method for producing fine grain sheet material for structural applications. In the CCGP process, a work-piece is repetitively bent and straightened without significantly changing the cross-section geometry of the work-piece, during which large plastic strains are imparted into the materials, which leads to the refinement of microstructure. Although many researchers worked on CCGP[10] but none of them study thoroughly on Metal matrix composite material under severe plastic deformation and CCGP process. In this present study the mechanical behavior were predicted through statistical analysis of the measured micro hardness at different conditions and find the effects of $\%$ of reinforcement, number of passes, strain rate, thickness of the plate and Heat treatment on properties of CCGP by using Taguchi $\mathrm{L}_{16}$ orthogonal array. The analysis of variance was employed to find the percentage of influence of various factors and its interaction on the physical behavior of CGP specimens.

\section{EXPERIMENTAL STUDIES}

In the present study, material selected for the composite preparation used $\mathrm{Al} 6061$ alloy. The chemical composition of Al 6061 alloy is 0.8-1.2 \% Magnesium, 0.4-0.8 \% Silicon, Max 0.7 \% Iron, 0.15-0.40\% Copper, Max $0.25 \%$ of Zinc, Max 0.15\% of Titanium, Max $0.15 \%$ of Manganese, 0.04-0.35\% Chromium and balanced $\%$ is Aluminum. Also $\mathrm{SiC}$ of particle size 30 to $50 \mu \mathrm{m}$ used as reinforcement. This was melted at $700^{\circ} \mathrm{C}$ which is slightly more than $30^{\circ} \mathrm{C}$ above the liquidus temperature. The dispersoid used was $\mathrm{SiC}$ particles of size 30$50 \mu \mathrm{m}$, fabricate the specimens, in which a vortex was created in the melt of the matrix alloy through a mechanical stirrer coated with aluminite and rotating at $550 \mathrm{rpm}$. The $\mathrm{SiC}$ particles were preheated to $200^{\circ} \mathrm{C}$ and added to the vortex of liquid melt at a rate of $120 \mathrm{~g} / \mathrm{min}$. A small amount of magnesium, which improves the wet ability of the $\mathrm{SiC}$ particles, was added along with the SiC.The composite melt was thoroughly stirred and subsequently degassed by passing nitrogen through at a rate of $2-3-1 / \min$ for 3 to 4 minutes. The composites 
were then cast in permanent moulds of dimension $20 \mathrm{~mm}$ width, $100 \mathrm{~mm}$ length and $10 \mathrm{~mm}$ thickness. $\mathrm{Al}$ alloy composites containing various $\mathrm{SiC}$ contents, namely $5 \%, 10 \%, 15 \%$ and $20 \%$ by weight were fabricated and tested and their properties were compared with those of the unreinforced matrix.From the as prepared composites plates the samples were cut with a width of $20 \mathrm{~mm}$ and length $100 \mathrm{~mm}$. The samples are taken in 4 different thicknesses, i.e., $3 \mathrm{~mm}, 4 \mathrm{~mm}, 5 \mathrm{~mm}$ and $6 \mathrm{~mm}$ having same length and width after machining. The $\mathrm{Al}$ alloy MMCs plates with above dimensions were pressed in a corrugated and flat die to produce ultra fine grain structures. These specimens were compressed using four different strain rates i.e. $0.5 \mathrm{~mm}, 1 \mathrm{~mm} / \mathrm{min}, 1.5$ $\mathrm{mm} / \mathrm{min}$ and $2 \mathrm{~mm} / \mathrm{min}$ and one to seven passes of compression were considered. The Vickers hardness (Hv) of the test specimens were calculated using Micromet-5101 device, with a load of 200g and loading period of 20 seconds. For obtaining reliable results, the measurements of the hardness was performed on all the specimens in three different locations and one among the two nearer value was considered as final hardness value.

\section{PLAN OF EXPERIMENTS}

Taguchi method is an efficient tool which enables the up gradation of the performance of the product, process, and design with significant prediction of cost and time. It is a systematic approach for enabling the design optimization there by ensuring both utility and performance by means of Taguchi's parameter design concept. The system performance could be optimized by means of systematic setting of design parameters and reducing the fluctuations. This method employs a special design of orthogonal arrays to study entire process parameters with small number of experiments $[11,12]$. Taguchi method based design experiment has been used to study effect of five CCGP process parameters i.e. Wt. \% of SiC, Strain rate, No. of passes, Thickness and Heat treatment on one important out put parameter micro hardness. Present study was focused on optimization of cyclic constrained groove pressing parameter on the based on higher strength. The paper was focused on effect of $\mathrm{SiC}$ particle, strain rate, number of passes, thickness and heat treatment conditions with four levels. Table 1 shows five factors and four levels used in the experiment. If four levels were assigned to each of these factors and a factorial experimental design was employed using each of these values, number of permutations would be 625 experiments. The fractional factorial design reduced the number of experiments to sixteen. Therefore Taguchi based $\mathrm{L}_{16}$ orthogonal array is selected shown in Table 1. CCGP process parameter and different levels are shown in table 2. Accordingly 16 experiments were carried out to study the effect of CCGP input parameters.

Table 1. Experimental layout using $\mathrm{L}_{16}$ orthogonal array

\begin{tabular}{|c|c|c|c|c|c|}
\hline \multirow{2}{*}{ Expt. No. } & \multicolumn{5}{|c|}{ Cyclic constrained groove pressing (CCGP) process parameters } \\
\cline { 2 - 6 } & $\mathbf{A}$ & $\mathbf{B}$ & $\mathbf{C}$ & $\mathbf{D}$ & $\mathbf{E}$ \\
\hline 1 & 5 & 0.5 & 1 & 3 & 0 \\
\hline 2 & 5 & 1.0 & 3 & 4 & 1 \\
\hline 3 & 5 & 1.5 & 5 & 5 & 3 \\
\hline 4 & 5 & 2.0 & 7 & 6 & 5 \\
\hline 5 & 10 & 0.5 & 3 & 5 & 5 \\
\hline 6 & 10 & 1.0 & 1 & 6 & 3 \\
\hline 7 & 10 & 1.5 & 7 & 3 & 1 \\
\hline 8 & 10 & 2.0 & 5 & 4 & 0 \\
\hline 9 & 15 & 0.5 & 5 & 6 & 1 \\
\hline 10 & 15 & 1.0 & 7 & 5 & 0 \\
\hline 11 & 15 & 1.5 & 1 & 4 & 5 \\
\hline 12 & 15 & 2.0 & 3 & 3 & 3 \\
\hline 13 & 20 & 0.5 & 7 & 4 & 3 \\
\hline 14 & 20 & 1.0 & 5 & 3 & 5 \\
\hline 15 & 20 & 1.5 & 3 & 6 & 0 \\
\hline 16 & 20 & 2.0 & 1 & 5 & 1 \\
\hline
\end{tabular}


Table 2. CCGP process parameters and different levels

\begin{tabular}{|c|c|l|l|l|l|l|}
\hline \multirow{2}{*}{ Symbol } & \multirow{2}{*}{ Factors } & \multirow{2}{*}{ Unit } & \multicolumn{5}{|c|}{ Levels } \\
\cline { 4 - 7 } & & & $\mathbf{1}$ & $\mathbf{2}$ & $\mathbf{3}$ & $\mathbf{4}$ \\
\hline $\mathrm{A}$ & $\mathrm{SiC}$ & $\mathrm{Wt} . \%$ & 5 & 10 & 15 & 20 \\
\hline B & Strain rate & $\mathrm{mm} / \mathrm{min}$ & 0.5 & 1.0 & 1.5 & 2.0 \\
\hline C & No. of passes & No. & 1 & 3 & 5 & 7 \\
\hline D & Thickness & mm & 3 & 4 & 5 & 6 \\
\hline E & Heat treatment & Hr & 0 & 1 & 3 & 5 \\
\hline
\end{tabular}

\section{DATA EVALUATION AND ANALYSIS}

Taguchi Methods use the S/N (signal-to-noise) ratio to analyses the test run results because the $\mathrm{S} / \mathrm{N}$ ratio represents both the average (mean) and variation (scatter) of the experimental results. The $\mathrm{S} / \mathrm{N}$ ratio is also used in Analysis of Variance (ANONA). The term $\mathrm{S} / \mathrm{N}$ ratio is borrowed from signal processing technology, but has different meanings here. A number of $\mathrm{S} / \mathrm{N}$ ratios are available in Taguchi Methods, e.g., smaller-the-better, larger-the-better, and nominal-the-best. The standard $\mathrm{S} / \mathrm{N}$ ratios can be customized to fit specific applications and new $\mathrm{S} / \mathrm{N}$ ratios can be developed for particular applications. Selecting the proper $\mathrm{S} / \mathrm{N}$ ratio depends on the physical properties of the problem, the engineering insight, the pursuing experiment results, etc.. In this study the Measurement of hardness is the objective function, so that the largerthe-better $\mathrm{S} / \mathrm{N}$ ratio is chosen. The basic formulas and notations used in this study can be found in reference [11].

$S / N_{\text {LTB }}=-10 \log [M S D]=-10 \log \left[\frac{1}{n} \sum_{i=1}^{n}\left(\frac{1}{y_{i}^{2}}\right)\right]$

Where $\mathrm{S} / \mathrm{N}_{\mathrm{LTB}}$ stands for larger-the-better Signal-to-Noise ratio, MSD is the mean square deviation around the target (infinity in this case), $\mathrm{y}_{\mathrm{i}}$ is the individually measured response value (experiment result), $\mathrm{n}$ is the number of measurements taken in one test run. Table 3 shows the results of each test run and the $S / \mathrm{N}_{\mathrm{LTB}}$ value calculated from equation (1).

The level average response analysis is based on averaging the experiment results achieved at each level for each parameter. In this study, each level for each parameter contains four test runs. It can be seen from Table 3 that while the level 1 of parameter A occurs, in test run 1, 2,3 and 4, all four levels of parameters B, C, D and E appear once in these four test runs. The level 2 of parameter A occurs in test run 5, 6, 7 and 8, whilst all four levels of parameter B, C, D and E also appear once in these four test runs. The level 3 of parameter A occurs in test run 9, 10,11 and 12, whilst all four levels of parameter B, C, D and E also appear once in these four test runs. The level 4 of parameter A is the same. Other levels of other parameters are the same, for example, level 1 of parameter B occurs at test runs 1, 5, 9 and 13, at these four test runs, all four levels of parameter A, C,D and E also appear once. When performing level average response analysis for one level of one parameter, all the influences from different levels of other parameters will be counterbalanced because every other parameter will appear at different level once. So the effect of one parameter at one level on the experiment results can be separated from other parameters. In this way, the effect of each level of every parameter can be viewed independently.

The level average response analysis is carried out by averaging the experimental results from three test runs corresponding to each level of each parameter, one by one, which is shown in Table 4 and plotted in Fig. 1.give the results of Level average response analysis for Micro hardness (VHN). Table 5 and Fig. 2 give the results of level average response analysis by $\mathrm{S} / \mathrm{N}$ ratio. 
Table 3 Experimental results

\begin{tabular}{|c|c|c|c|c|c|c|c|c|c|c|c|}
\hline \multirow[t]{2}{*}{$\begin{array}{l}\text { Ex. } \\
\text { No. }\end{array}$} & \multicolumn{5}{|c|}{ Main factors } & \multicolumn{3}{|c|}{$\begin{array}{c}\text { Observed response (Micro } \\
\text { Hardness)VHN }\end{array}$} & \multirow[t]{2}{*}{$\begin{array}{l}\text { Average } \\
\text { VHN }\end{array}$} & \multirow[t]{2}{*}{$\begin{array}{l}\text { Standard } \\
\text { Deviation }\end{array}$} & \multirow[t]{2}{*}{$\begin{array}{c}\mathrm{S} / \mathrm{N} \\
\text { ratio }\end{array}$} \\
\hline & $\mathbf{A}$ & B & $\mathbf{C}$ & D & $\mathbf{E}$ & 1 & 2 & 3 & & & \\
\hline 1 & 5 & 0.5 & 1 & 3 & 0 & 53.19 & 54.61 & 50.84 & 52.88 & 1.904 & 34.46 \\
\hline 2 & 5 & 1.0 & 3 & 4 & 1 & 75.29 & 77.83 & 72.76 & 75.29 & 2.535 & 37.53 \\
\hline 3 & 5 & 1.5 & 5 & 5 & 3 & 95.91 & 97.07 & 94.08 & 95.69 & 1.507 & 39.62 \\
\hline 4 & 5 & 2.0 & 7 & 6 & 5 & 117.28 & 120.18 & 114.65 & 117.37 & 2.766 & 41.39 \\
\hline 5 & 10 & 0.5 & 3 & 5 & 5 & 118.05 & 120.01 & 117.29 & 118.45 & 1.403 & 41.47 \\
\hline 6 & 10 & 1.0 & 1 & 6 & 3 & 106.30 & 107.34 & 105.73 & 106.46 & 0.816 & 40.54 \\
\hline 7 & 10 & 1.5 & 7 & 3 & 1 & 114.83 & 115.70 & 114.54 & 115.02 & 0.604 & 41.21 \\
\hline 8 & 10 & 2.0 & 5 & 4 & 0 & 112.07 & 114.42 & 109.68 & 112.06 & 2.370 & 40.99 \\
\hline 9 & 15 & 0.5 & 5 & 6 & 1 & 140.72 & 141.84 & 140.23 & 140.93 & 0.825 & 42.98 \\
\hline 10 & 15 & 1.0 & 7 & 5 & 0 & 141.96 & 143.83 & 141.61 & 142.47 & 1.194 & 43.07 \\
\hline 11 & 15 & 1.5 & 1 & 4 & 5 & 140.77 & 142.99 & 138.98 & 140.91 & 2.009 & 42.98 \\
\hline 12 & 15 & 2.0 & 3 & 3 & 3 & 139.98 & 140.39 & 139.07 & 139.81 & 0.676 & 42.91 \\
\hline 13 & 20 & 0.5 & 7 & 4 & 3 & 176.30 & 177.86 & 175.65 & 176.60 & 1.136 & 44.94 \\
\hline 14 & 20 & 1.0 & 5 & 3 & 5 & 176.36 & 177.32 & 175.02 & 176.23 & 1.155 & 44.92 \\
\hline 15 & 20 & 1.5 & 3 & 6 & 0 & 163.00 & 164.43 & 161.42 & 162.95 & 1.506 & 44.24 \\
\hline 16 & 20 & 2.0 & 1 & 5 & 1 & 157.63 & 160.25 & 155.65 & 157.84 & 2.307 & 43.96 \\
\hline
\end{tabular}

Table 4 Level average response analysis for Micro hardness (Hv)

\begin{tabular}{|c|c|c|c|c|c|c|c|c|c|c|c|}
\hline & $\begin{array}{l}\text { Test } \\
\text { Run } \\
\text { No. }\end{array}$ & $\begin{array}{l}\text { Average of } \\
\text { each nun } \\
\text { (VHN) }\end{array}$ & $\begin{array}{c}\text { Level Average } \\
\text { reaponse } \\
\text { Micro } \\
\text { hardiness } \\
\text { (VHD) }\end{array}$ & & \begin{tabular}{l|} 
Test \\
Run \\
No.
\end{tabular} & $\begin{array}{l}\text { Average of } \\
\text { each nun } \\
\text { (VHN) }\end{array}$ & $\begin{array}{l}\text { Level } \\
\text { Average } \\
\text { reaponise } \\
\text { Micro } \\
\text { hardness } \\
\text { (VHN) }\end{array}$ & & $\begin{array}{l}\text { Test } \\
\text { Run } \\
\text { No. }\end{array}$ & $\begin{array}{c}\text { Average of } \\
\text { each nun } \\
\text { (VHN) }\end{array}$ & $\begin{array}{c}\text { Level } \\
\text { Average } \\
\text { response } \\
\text { Micro } \\
\text { hardinass } \\
\text { (VHN) }\end{array}$ \\
\hline \multicolumn{4}{|c|}{ Parameter $\mathrm{A}: \mathrm{SiC} \%$} & \multicolumn{4}{|c|}{ Parameter C: No. of passes } & \multicolumn{4}{|c|}{ Parameter E: Heat treatment } \\
\hline \multirow{4}{*}{$\begin{array}{l}\text { Level } 1 \text {, } \\
5 \%\end{array}$} & 1 & 52.88 & \multirow{4}{*}{85.31} & \multirow{4}{*}{$\begin{array}{l}\text { Levell, } \\
5 \%\end{array}$} & 1 & 52.88 & \multirow{4}{*}{114.52} & \multirow{4}{*}{$\begin{array}{l}\text { Levell, } \\
5 \%\end{array}$} & 1 & 52.88 & \multirow{4}{*}{117.59} \\
\hline & 2 & 75.29 & & & 6 & 106.46 & & & 8 & 112.06 & \\
\hline & 3 & 95.69 & & & 11 & 140.91 & & & 10 & 142.47 & \\
\hline & 4 & 117.37 & & & 16 & 157.84 & & & 15 & 162.95 & \\
\hline \multirow{4}{*}{$\begin{array}{l}\text { Level } 2 \text {, } \\
10 \%\end{array}$} & 5 & 118.45 & \multirow{4}{*}{113.00} & \multirow{4}{*}{$\begin{array}{l}\text { Level } 2 \text {, } \\
10 \% \%\end{array}$} & 2 & 75.29 & \multirow{4}{*}{124.13} & \multirow{4}{*}{$\begin{array}{l}\text { Leval } 2, \\
10 \%\end{array}$} & 2 & 75.29 & \multirow{4}{*}{122.27} \\
\hline & 6 & 106.46 & & & 5 & 118.45 & & & 7 & 115.02 & \\
\hline & 7 & 115.02 & & & 12 & 139.81 & & & 9 & 140.93 & \\
\hline & 8 & 112.06 & & & 15 & 162.95 & & & 16 & 157.84 & \\
\hline \multirow{4}{*}{$\begin{array}{l}\text { Level 3, } \\
15 \%\end{array}$} & 9 & 140.93 & \multirow{4}{*}{141.03} & \multirow{4}{*}{$\begin{array}{l}\text { Level 3, } \\
15 \%\end{array}$} & 3 & 95.69 & \multirow{4}{*}{131.23} & \multirow{4}{*}{$\begin{array}{l}\text { Level 3, } \\
15 \%\end{array}$} & 3 & 95.69 & \multirow{4}{*}{129.64} \\
\hline & 10 & 142.47 & & & 8 & 112.06 & & & 6 & 106.46 & \\
\hline & 11 & 140.91 & & & 9 & 140.93 & & & 12 & 139.81 & \\
\hline & 12 & 139.81 & & & 14 & 176.23 & & & 13 & 176.60 & \\
\hline \multirow{4}{*}{$\begin{array}{l}\text { Level } 4, \\
20 \% \%\end{array}$} & 13 & 176.60 & \multirow{4}{*}{168.41} & \multirow{4}{*}{$\begin{array}{l}\text { Level } 4, \\
20 \%\end{array}$} & 4 & 117.37 & \multirow{4}{*}{137.87} & \multirow{4}{*}{$\begin{array}{l}\text { Lavel 4, } \\
20 \%\end{array}$} & 4 & 117.37 & \\
\hline & 14 & 176.23 & & & 7 & 115.02 & & & 5 & 118.45 & \\
\hline & 15 & 162.95 & & & 10 & 142.47 & & & 11 & 140.91 & 132.55 \\
\hline & 16 & 157.84 & & & 13 & 176.60 & & & 14 & 176.23 & \\
\hline Paramete & B: Strai & rate & & Parameter & D: Thic & kness & & & & & \\
\hline & 1 & 52.88 & & & 1 & 52.88 & & & & & \\
\hline Level 1 , & 5 & 118.45 & 1020 & Level 1 , & 7 & 115.02 & & & & & \\
\hline $5 \%$ & 9 & 140.93 & 122.22 & $5 \%$ & 12 & 139.81 & 120.99 & & & & \\
\hline & 13 & 176.60 & & & 14 & 176.23 & & & & & \\
\hline & 2 & 75.29 & & & 2 & 75.29 & & & & & \\
\hline Lavel 2, & 6 & 106.46 & & Level 2 , & 8 & 112.06 & & & & & \\
\hline $10 \%$ & 10 & 142.47 & 125.11 & $10 \%$ & 11 & 140.91 & 126.22 & & & & \\
\hline & 14 & 176.23 & & & 13 & 176.60 & & & & & \\
\hline & 3 & 95.69 & & & 3 & 95.69 & & & & & \\
\hline Level 3, & 7 & 115.02 & 128.64 & Lavel 3, & 5 & 118.45 & & & & & \\
\hline $15 \%$ & 11 & 140.91 & 128.64 & $15 \%$ & 10 & 142.47 & 128.01 & & & & \\
\hline & 15 & 162.95 & & & 16 & 157.84 & & & & & \\
\hline & 4 & 117.37 & & & 4 & 117.37 & & & & & \\
\hline Level 4, & 8 & 112.06 & 1317 & Lavel 4, & 6 & 106.46 & 13103 & & & & \\
\hline $20 \%$ & 12 & 139.81 & 151.7 & $20 \%$ & 9 & 140.93 & 131.92 & & & & \\
\hline & 16 & 157.84 & & & 15 & 162.95 & & & & & \\
\hline
\end{tabular}


Fig. 1 Level average response analysis for Micro hardness (VHN)

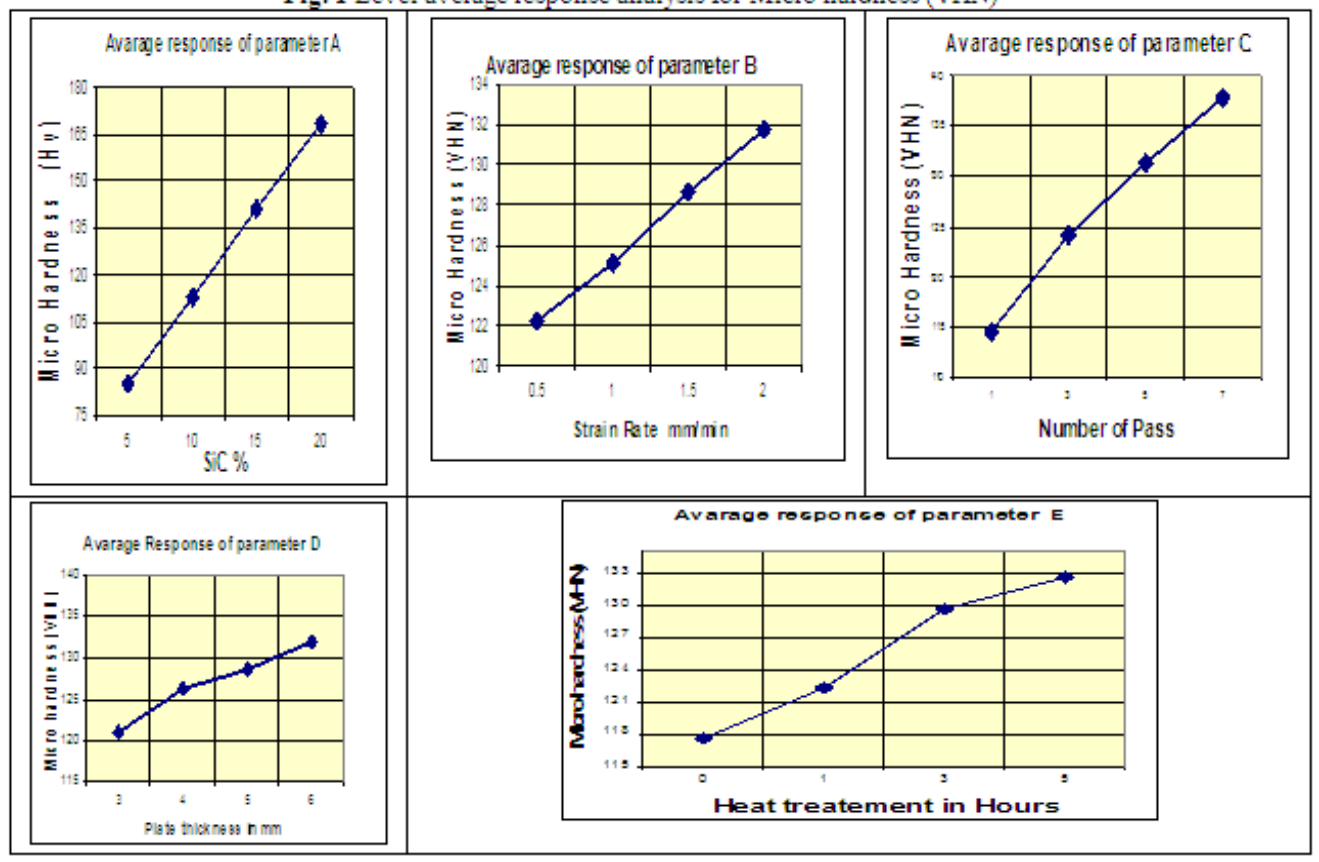

Table 5 Level average response analysis using $\mathrm{S} / \mathrm{N}_{\mathrm{LTB}}$ Ratio

\begin{tabular}{|c|c|c|c|c|c|c|c|c|c|c|c|}
\hline & $\begin{array}{c}\text { Tes } \\
\mathrm{t} \\
\mathrm{Ru} \\
\mathrm{n} \\
\text { No. }\end{array}$ & $\begin{array}{l}\text { S/NLT } \\
\text { B Ratio } \\
\text { of each } \\
\text { run } \\
\text { (dB) }\end{array}$ & $\begin{array}{c}\text { Level } \\
\text { Avera } \\
\text { ge } \\
\text { S/NLT } \\
\text { B (dB) }\end{array}$ & & $\begin{array}{l}\text { Test } \\
\text { Run } \\
\text { No. }\end{array}$ & $\begin{array}{c}\text { S/NLT } \\
\text { B Ratio } \\
\text { of each } \\
\text { run } \\
\text { (dB) }\end{array}$ & $\begin{array}{c}\text { Level } \\
\text { Average } \\
\text { S/NLTB } \\
(\mathrm{dB})\end{array}$ & & $\begin{array}{c}\text { Tes } \\
\mathrm{t} \\
\mathrm{Ru} \\
\mathrm{n} \\
\text { No. }\end{array}$ & $\begin{array}{c}\text { S/NLT } \\
\text { B Ratio } \\
\text { of each } \\
\text { run } \\
\text { (dB) }\end{array}$ & $\begin{array}{c}\text { Lev } \\
\text { el } \\
\text { Ave } \\
\text { rage } \\
\text { S/N } \\
\text { LTB } \\
\text { (dB) }\end{array}$ \\
\hline \multicolumn{4}{|c|}{ Parameter A: SiC \% } & \multicolumn{4}{|c|}{ Parameter C: No. of passes } & \multicolumn{4}{|c|}{$\begin{array}{l}\text { Parameter E: Heat } \\
\text { treatment }\end{array}$} \\
\hline \multirow{4}{*}{$\begin{array}{l}\text { Leve } \\
11 \\
5 \%\end{array}$} & 1 & 34.46 & \multirow{4}{*}{38.25} & \multirow{4}{*}{$\begin{array}{l}\text { Level } \\
1,5 \%\end{array}$} & 1 & 34.4 & \multirow{4}{*}{40.47} & \multirow{4}{*}{$\begin{array}{l}\text { Leve } \\
11, \\
5 \%\end{array}$} & 1 & 34.4 & \multirow{4}{*}{$\begin{array}{c}40.6 \\
7\end{array}$} \\
\hline & 2 & 37.53 & & & 6 & 40.54 & & & 8 & 40.99 & \\
\hline & 3 & 39.62 & & & 11 & 42.98 & & & 10 & 43.07 & \\
\hline & 4 & 41.39 & & & 16 & 43.96 & & & 15 & 44.24 & \\
\hline \multirow{4}{*}{$\begin{array}{l}\text { Leve } \\
12, \\
10 \%\end{array}$} & 5 & 41.47 & \multirow{4}{*}{41.05} & \multirow{4}{*}{$\begin{array}{l}\text { Level } \\
2, \\
10 \%\end{array}$} & 2 & 37.53 & \multirow{4}{*}{41.54} & \multirow{4}{*}{$\begin{array}{l}\text { Leve } \\
12, \\
10 \%\end{array}$} & 2 & 37.53 & \multirow{4}{*}{$\begin{array}{c}41.4 \\
2\end{array}$} \\
\hline & 6 & 40.54 & & & 5 & 41.47 & & & 7 & 41.21 & \\
\hline & 7 & 41.21 & & & 12 & 42.91 & & & 9 & 42.98 & \\
\hline & 8 & 40.99 & & & 15 & 44.24 & & & 16 & 43.96 & \\
\hline \multirow{4}{*}{$\begin{array}{l}\text { Leve } \\
13 \text {, } \\
15 \%\end{array}$} & 9 & 42.98 & \multirow{4}{*}{42.98} & \multirow{4}{*}{$\begin{array}{l}\text { Level } \\
3, \\
15 \%\end{array}$} & 3 & 39.62 & \multirow{4}{*}{42.13} & \multirow{4}{*}{$\begin{array}{l}\text { Leve } \\
13 \\
15 \%\end{array}$} & 3 & 39.62 & \multirow{4}{*}{$\begin{array}{c}42.0 \\
0\end{array}$} \\
\hline & 10 & 43.07 & & & 8 & 40.99 & & & 6 & 40.54 & \\
\hline & 11 & 42.98 & & & 9 & 42.98 & & & 12 & 42.91 & \\
\hline & 12 & 42.91 & & & 14 & 44.92 & & & 13 & 44.94 & \\
\hline \multirow{4}{*}{$\begin{array}{l}\text { Leve } \\
14, \\
20 \%\end{array}$} & 13 & 44.94 & \multirow{4}{*}{44.51} & \multirow{4}{*}{$\begin{array}{l}\text { Level } \\
4, \\
20 \%\end{array}$} & 4 & \begin{tabular}{|l|}
41.39 \\
\end{tabular} & \multirow{4}{*}{42.65} & \multirow{4}{*}{$\begin{array}{l}\text { Leve } \\
14, \\
20 \%\end{array}$} & 4 & 41.39 & \\
\hline & 14 & 44.92 & & & 7 & 41.21 & & & 5 & 41.47 & 42.6 \\
\hline & 15 & 44.24 & & & 10 & 43.07 & & & 11 & 42.98 & 9 \\
\hline & 16 & 43.96 & & & 13 & 44.94 & & & 14 & 44.92 & \\
\hline Parai & eter I & : Strain I & ate & Para & er D: & Thickness & & & & & \\
\hline & 1 & 34.4 & & & 1 & 34.4 & & & & & \\
\hline Leve & 5 & 41.47 & 4005 & Level & 7 & 41.21 & 1086 & & & & \\
\hline 11, & 9 & 42.98 & 40.95 & $1,5 \%$ & 12 & 42.91 & 40.86 & & & & \\
\hline & 13 & 44.94 & & & 14 & 44.92 & & & & & \\
\hline & 2 & 37.53 & & & 2 & 37.53 & & & & & \\
\hline Leve & 6 & 40.54 & 4152 & Level & 8 & 40.99 & 4161 & & & & \\
\hline 12, & 10 & 43.07 & 41.52 & & 11 & 42.98 & 41.61 & & & & \\
\hline & 14 & 44.92 & & & 13 & 44.94 & & & & & \\
\hline & 3 & 39.62 & & & 3 & 39.62 & & & & & \\
\hline Leve & 7 & 41.21 & 12 & Level & 5 & 41.47 & 4202 & & & & \\
\hline $\begin{array}{l}13, \\
15 \%\end{array}$ & 11 & 42.98 & 42.01 & & 10 & 43.07 & 42.03 & & & & \\
\hline & 15 & 44.24 & & & 16 & 43.96 & & & & & \\
\hline & 4 & 41.39 & & & 4 & \begin{tabular}{|l|}
41.39 \\
\end{tabular} & & & & & \\
\hline Leve & 8 & 40.99 & 423 & Level & 6 & 40.54 & $42 \pi$ & & & & \\
\hline 14, & 12 & 42.91 & 42.31 & 4, & 9 & 42.98 & 42.29 & & & & \\
\hline & 16 & 43.96 & & & 15 & 44.24 & & & & & \\
\hline
\end{tabular}


Fig. 2 level average response analysis by $\mathrm{S} / \mathrm{N}$ ratio.

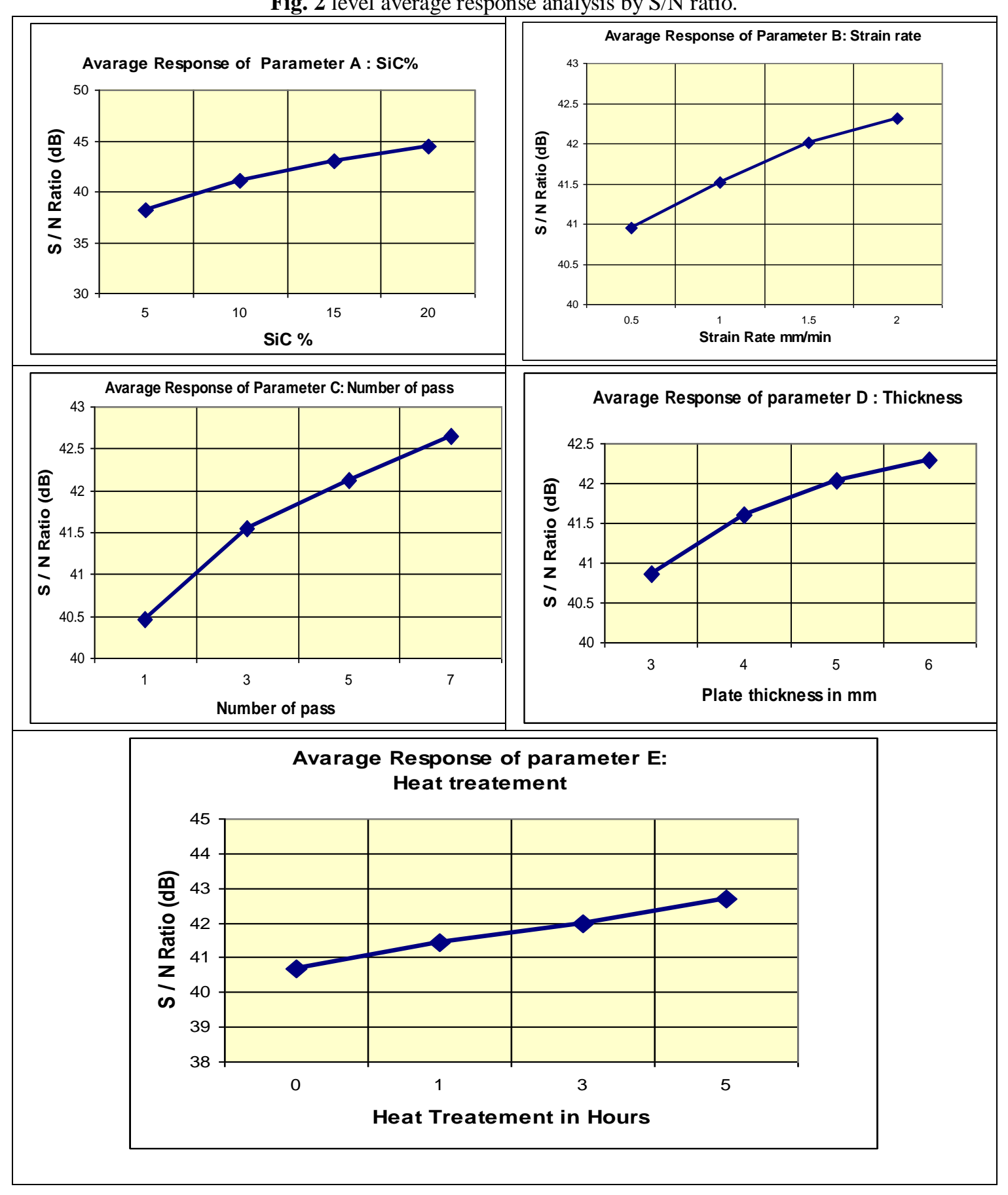

\section{Analysis Of Variance (ANOVA)}

\section{RESULT AND DISCUSSION}

Analysis of variance is a computational technique to quantitatively estimate the relative contribution, which each controlled parameter makes to the overall measured response and expressing it as a percentage. Thus the information about how significant the effect of each controlled parameter is on the experimental results can be obtained. ANOVA uses $\mathrm{S} / \mathrm{N}$ ratio responses to calculate. The basic idea of ANOVA is $\sigma_{\mathrm{T}}^{2}=\sigma_{\mathrm{A}}^{2}+\sigma_{\mathrm{B}}{ }^{2}+$ $\sigma_{\mathrm{C}}^{2}+\sigma_{\mathrm{D}}^{2}+\sigma_{\mathrm{E}}^{2}$, that the total sum of the squares of the standard deviation $\left(\sigma_{\mathrm{T}}^{2}\right.$, total variation) is equal to sum of the squares of the standard deviation caused by each parameter $\sigma_{\mathrm{A}}{ }^{2}, \sigma_{\mathrm{B}}{ }^{2}, \sigma_{\mathrm{C}}{ }^{2}, \sigma_{\mathrm{D}}{ }^{2}, \sigma_{\mathrm{E}}{ }^{2}$ The overall mean from which all the variation (standard deviation) is calculated is given by

$$
\overline{S / N}=\frac{1}{n} \sum_{i=1}^{n} S / N_{i}
$$




$$
\overline{S / N}_{L T B}=\frac{1}{16} \sum_{i=1}^{16}\left(S / N_{L T B}\right)_{i}=\frac{1}{16} \begin{gathered}
{[34.46+37.53+39.62+41.39+} \\
+42.98+43.07+42.98+42.91
\end{gathered}
$$

\section{$\begin{aligned} & 41.47+40.54+41.21+40.99 \\ & +44.94+44.92+44.24+43.96]\end{aligned}=\mathbf{4 1 . 6 9}$}

The grand total sum of squares GTSS is given by In this case,

$$
\begin{aligned}
& G T S S=\sum_{i=1}^{n}\left(S / N_{i}\right)^{2} \\
& \text { GTSS }=\sum_{i=1}^{16}\left(S / N_{i}\right)^{2}= \\
& {\left[34.46^{\mathbf{2}}+37.53^{\mathbf{2}}+39.62+41.39^{\mathbf{2}}+41.47^{\mathbf{2}}+40.54^{\mathbf{2}}+41.21+40.99^{\mathbf{2}}+\right.} \\
& \left.+42.98^{\mathbf{2}}+43.07+42.98+42.91+44.94^{\mathbf{2}}+44.92+44.24+43.96\right] \\
& \mathbf{2}
\end{aligned}
$$

The GTSS can be decomposed into two parts, the sum of the squares due to overall mean and the sum of the squares due to variation around overall mean:

$$
\mathrm{GTSS}=\mathrm{SS}_{\text {mean }}+\mathrm{SS}_{\text {variation }}
$$

1. The sum of the squares due to overall mean:

$$
S S_{\text {mean }}=n \times(\overline{S / N})^{2}
$$

Where $\mathrm{n}$ is the number of total test runs In this case,

$$
\begin{aligned}
& S S_{\text {mean }}=16 \times(\overline{S / N})^{2} \\
& S S_{\text {mean }}=16 \times(41.69)^{2}=27808.89
\end{aligned}
$$

2. The sum of the squares due to variation around overall mean:

$$
S S_{\text {variation }}=\frac{1}{n} \sum_{i=1}^{n}\left(S / N_{i}-\overline{S / N}\right)^{2}
$$

In this study,

$$
S S_{\text {variation }}=\sum_{i=1}^{16}\left(\left(S / N_{L T B}\right)_{i}-\overline{S / N}_{L T B}\right)^{2}
$$


In this study,

$$
\begin{array}{r}
\mathrm{SS}_{\text {variation }}=(34.46-41.69)^{2}+(37.53-41.69)^{2}+(39.62-41.69)^{2}+(41.39-41.69)^{2}+ \\
(41.47-41.69)^{2}+(40.54-41.69)^{2}+(41.21-41.69)^{2}+(40.99-41.69)^{2}+ \\
(42.98-41.69)^{2}+(43.07-41.69)^{2}+(42.98-41.69)^{2}+(42.91-41.69)^{2}+ \\
\mathrm{SS}_{\text {variation }}=115.41
\end{array}
$$

The SSvariation can be further decomposed into the sums of the squares of the variation induced by individual parameter effects around overall mean. From The level average response analysis table, it is known that when doing level average response analysis for one level of one parameter, all the influences from different levels of other parameters will be counterbalanced. So, the level average $\mathrm{S} / \mathrm{N}$ minus overall mean $\mathrm{S} / \mathrm{N}$ is the variation caused by that parameter at that level.

For parameter A, the sum of the squares due to variation around overall mean is

$$
\mathrm{SS}_{A}=n_{A 1} \times\left(\overline{S / N}_{A 1}-\overline{S / N}\right)^{2}+n_{A 2} \times\left(\overline{S / N}_{A 2}-\overline{S / N}\right)^{2}+n_{A 3} \times\left(\overline{S / N}_{A 3}-\overline{S / N}^{2}+n_{A 4} \times\left(\overline{S / N}_{A 4}-\overline{S / N}\right)^{2}\right.
$$

In this case,

$\mathrm{SS}_{\mathrm{A}}=4 \mathrm{x}(38.25-41.69) 2+4 \mathrm{x}(41.05-41.69) 2+4 \mathrm{x}(42.98-41.69) 2+4 \mathrm{x}(44.51-41.69) 2=87.41$

Similarly,

$\mathrm{SS}_{\mathrm{B}}=4 \times(40.95-41.69) 2+4 \times(41.51-41.69) 2+4 \times(42.01-41.69) 2+4 \times(42.31-41.69) 2=4.27$

$\mathrm{SS}_{\mathrm{C}}=4 \times(40.47-41.69) 2+4 \times(41.54-41.69) 2+4 \times(42.13-41.69) 2+4 \times(42.65-41.69) 2=10.50$

$\mathrm{SS}_{\mathrm{D}}=4 \times(40.86-41.69) 2+4 \times(41.61-41.69) 2+4 \times(42.03-41.69) 2+4 \times(42.29-41.69) 2=4.68$

$\mathrm{SSE}=4 \times(40.68-41.69) 2+4 \times(41.42-41.69) 2+4 \times(42.00-41.69) 2+4 \times(42.69-41.69) 2=8.76$

The percentage contribution of each parameter is found:

\section{Percentage contribution of Parameter $=($ SSparameter $) /($ SSvariation $)$}

In this study,

Parameter A, SiC \%:

$(87.41 / 115.41) \times 100=\mathbf{7 5 . 7 4 \%}$

Parameter B: Strain rate:

$(4.27 / 115.41) \times 100=\mathbf{3 . 7 0 \%}$

Parameter C: No. of passes:

$(10.50 / 115.41) \times 100=\mathbf{9 . 1 \%}$

Parameter D: Thickness of the plate:

$(4.68 / 115.41) \times 100=\mathbf{4 . 0 5 \%}$

Parameter E: Heat treatment:

$(8.76 / 115.41) \times 100=\mathbf{7 . 5 9 \%}$

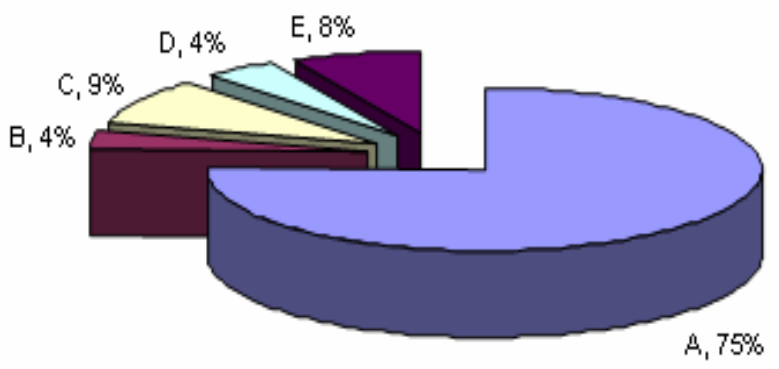

$\square A \square B \square C \square D \square E$

Fig.3 Percentage contributions for CCGP parameters A, B, C, D, and E by ANOVA.

The ANOVA has been performed to predict the statistical significance of the process parameters. It helps to determine the effect of individual input parameter on output parameters. The results of ANOVA presented for CCGP in percentage contribution $(\mathrm{P})$ of each factor on the total variation with their degree of influence on the result. It can be observed from calculation that the reinforcement Sic $(\mathrm{A}=$ 
$75.74 \%)$, Strain rate $(\mathrm{B}=3.7 \%)$, Number of pass $(\mathrm{C}=9.1 \%)$, thickness of the plate $(\mathrm{D}=4.05 \%)$ and Heat treatment $(\mathrm{E}=7.59 \%)$ The pooled error is $0.009 \%$. Based on the results presented in calculations, reinforcement of Sic is found to be the most influencing process parameter with contribution followed by number of pass, heat treatment, plate thickness and strain rate and this apportionment shown in Fig. 3. The main effects plot generated by excel sheet pertaining to ANOVA. The ranking of process parameters generated by the conduct of Taguchi method is shown in Table.5. As inferred from Table 5, reinforcement of Sic has been ranked as the highly prioritized process parameter followed by Number of pass, Heat treatment, the plate thickness and strain rate for the cyclic constrained groove pressing process.

\section{CONCLUSION}

* Taguchi Methods have been successfully applied to AL/Sic composite plate subjected to CCGP process parameter optimization in the first step of pressing

* From the Experiments it is clear that the parameter reinforcement of $\mathrm{SiC}$ (A) has a highest amount of contribution on response values, Number of passes (C) has the second highest contribution on the response values and Heat treatment $(E)$ the third highest contribution on the response values.

* Other values of the thickness (D) and strain rate (B) show a very less amount of contribution.

- From the Taguchi method it is confirmed that the best combination of experiment run which has a highest order and gives highest values on all the response values is experiment number 13

- $\mathrm{Al}$ alloy composites containing $\mathrm{SiC}$ of $20 \%$ by weight, For $4 \mathrm{~mm}$ thick $\mathrm{Al} / \mathrm{Sic}$ composite plate compressed by $0.5 \mathrm{~mm} / \mathrm{min}$ strain rate up to7 passes and heat treated foe 3hours gives the highest hardness $176.6 \mathrm{Hv}$.

* From the Taguchi analysis it is concluded that, at 99\% confidence level, Al alloy composites containing $\mathrm{SiC}$ has the highest contribution toward the response values obtained and the number of passes occupies the second highest contribution rate.

\section{REFERENCES}

[1]. R. Z. Valiev, I. V. Alexandrov, "NanostructuredMaterialsProduced by, Severe Plastic Deformation" Logos, Moscow, 2000.

[2]. T. C. Lowe, R. Z. Valiev (Eds.), "Investigation and Applications of Severe Plastic Deformation" Kluwer, Dodrecht, 2000.

[3]. Z. Horita, T Fujinami, M Nemoto, T G Langdon,'Improvement of mechanical properties for Al alloys using equal-channel angular pressing," Journal of Materials Processing Technology, vol. 117 (2001), pp.288-292.

[4]. V.M. Segal, "Materials processing by simple shear", Mater. Sci. Eng. A197 (1995) 157-164.

[5]. N.Tsuji,K.Shiotsuki, H. Utsunomiya, and Y. Saito, in Proceedings of the International Symposiumon"Towards Innovation in Superplasticity-"TransTech.publications.

[6]. Krishnaiah A, Chakkingal U, Venugopal P. Production of ultrafine grain sizes in aluminium sheets by severe plastic deformation using the technique of groove pressing. Scripta Mater 2005;52:122933.

[7]. Z. Horita, M. Furukawa, K. Oh-Ishi, M. Nemoto, T.G. Langdon, in: T. Saki, H.G. Suzuki (Eds.), Proceeding of the fourth International Conference on Recystallization and Related Phenomena, Japan Institute of Metals, Japan, 1999, p. 301.

[8]. J. Gill Sevillano, P. van Houtte, E. Aernoudt, Prog. Mater. Sci. 25 (1980) 69.

[9]. Huang JY, Zhu YT, Jiang H, Lowe TC. Microstructures and dislocation configurations in nanostructured cu processed BY repetitive corrugation and straightening, Acta Mater, vol.49 (2001), pp. 1497-505.

[10]. S. V. Dobatkin, "Severe Plastic Deformation of Steels: Structure, Properties and Techniques"in Investigation and Applications of Severe Plastic Deformation,Ed. by T. C. Lowe and R. Valiev (Kluwer, Netherlands), 3 (2000) 13-22.

[11]. Cochran, W. G. and Cox, G. M. Experimental designs, 2nd 1968,(Wiley, New York; London).

[12]. Fowlkes, W. Y. and Creveling, C. M., Engineering methods for robust product design, Using Taguchi Methods in technology and product development 1995,(Addison-Wesley Publishing Company). 